\title{
Algebraic setup of non-strict multiple zeta values
}

by

\author{
Shuichi Muneta (Fukuoka)
}

1. Introduction. The multiple zeta values and non-strict multiple zeta values (MZVs and NMZVs, for short) are defined respectively by

$$
\begin{aligned}
& \zeta\left(k_{1}, \ldots, k_{n}\right):=\sum_{m_{1}>\cdots>m_{n}>0} \frac{1}{m_{1}^{k_{1}} \cdots m_{n}^{k_{n}}}, \\
& \bar{\zeta}\left(k_{1}, \ldots, k_{n}\right):=\sum_{m_{1} \geq \cdots \geq m_{n}>0} \frac{1}{m_{1}^{k_{1}} \cdots m_{n}^{k_{n}}},
\end{aligned}
$$

where $k_{1}, \ldots, k_{n}$ are positive integers and $k_{1} \geq 2$. Considerable amount of work on MZVs has been done from various points of view.

There are several relations among the MZVs (duality formula, sum formula, Hoffman's relations, Ohno's relations, derivation relations and cyclic sum relations, cf. [2], [4], [6], [8]), and these relations can be described in a purely algebraic manner (cf. [6]). On the other hand, NMZVs have not been investigated so much compared to MZVs. But recently, a few works on NMZVs have appeared ([1], [9]) and they indicate that NMZVs have similar properties to MZVs.

In this article, we introduce an algebraic setup of NMZVs and use it to prove some relations among them, which are analogous to Hoffman's relations for MZVs.

\section{Algebraic setup of NMZVs}

2.1. Algebraic setup of $M Z V s$. We summarize the algebraic setup of MZVs introduced by Hoffman (cf. [3], [6]). Let $\mathfrak{H}=\mathbb{Q}\langle x, y\rangle$ be the noncommutative polynomial ring in two indeterminates $x, y$, and $\mathfrak{H}^{1}$ and $\mathfrak{H}^{0}$ its subrings $\mathbb{Q}+\mathfrak{H} y$ and $\mathbb{Q}+x \mathfrak{H} y$. We set $z_{k}=x^{k-1} y(k=1,2, \ldots)$. Then $\mathfrak{H}^{1}$ 
is freely generated by $\left\{z_{k}\right\}_{k \geq 1}$. For any word $w$, let $l(w)$ be the degree of $w$ with respect to $y$, and $|w|$ the total degree.

We define the $\mathbb{Q}$-linear map (called the evaluation map) $Z: \mathfrak{H}^{0} \rightarrow \mathbb{R}$ by

$$
Z(1)=1 \quad \text { and } Z\left(z_{k_{1}} \cdots z_{k_{n}}\right)=\zeta\left(k_{1}, \ldots, k_{n}\right) .
$$

We next define two products of MZVs. The one is the harmonic product * on $\mathfrak{H}^{1}$ defined by

$$
\begin{aligned}
1 * w & =w * 1=w \\
z_{k} w_{1} * z_{l} w_{2} & =z_{k}\left(w_{1} * z_{l} w_{2}\right)+z_{l}\left(z_{k} w_{1} * w_{2}\right)+z_{k+l}\left(w_{1} * w_{2}\right)
\end{aligned}
$$

$\left(k, l \in \mathbb{Z}_{\geq 1}\right.$ and $w, w_{1}, w_{2}$ are words in $\left.\mathfrak{H}^{1}\right)$, extended by $\mathbb{Q}$-bilinearity. The harmonic product $*$ is commutative and associative, therefore $\mathfrak{H}^{1}$ is a commutative $\mathbb{Q}$-algebra with respect to $*$. We denote it by $\mathfrak{H}_{*}^{1}$. The subset $\mathfrak{H}^{0}$ is a subalgebra of $\mathfrak{H}^{1}$ with respect to $*$ and we denote it by $\mathfrak{H}_{*}^{0}$. We then have

$$
Z\left(w_{1} * w_{2}\right)=Z\left(w_{1}\right) Z\left(w_{2}\right) \quad \text { for any } w_{1}, w_{2} \in \mathfrak{H}^{0} .
$$

The other product is the shuffle product $\mathrm{m}$ on $\mathfrak{H}$ defined by

$$
\begin{aligned}
1 ш w & =w ш 1=w, \\
u_{1} w_{1} \amalg u_{2} w_{2} & =u_{1}\left(w_{1} \amalg u_{2} w_{2}\right)+u_{2}\left(u_{1} w_{1} \amalg w_{2}\right)
\end{aligned}
$$

$\left(u_{1}, u_{2} \in\{x, y\}\right.$ and $w, w_{1}, w_{2}$ are words in $\left.\mathfrak{H}\right)$, extended by $\mathbb{Q}$-bilinearity. The shuffle product $ш$ is also commutative and associative, so $\mathfrak{H}$ is a commutative $\mathbb{Q}$-algebra with respect to $ш$. We denote it by $\mathfrak{H}_{\text {шा }}$. The subsets $\mathfrak{H}^{1}$ and $\mathfrak{H}^{0}$ are subalgebras of $\mathfrak{H}$ with respect to $ш$ and we denote them by $\mathfrak{H}_{\mathrm{II}}^{1}$, $\mathfrak{H}_{\text {III }}^{0}$ respectively. For this product, we also have

$$
Z\left(w_{1} \amalg w_{2}\right)=Z\left(w_{1}\right) Z\left(w_{2}\right) \quad \text { for any } w_{1}, w_{2} \in \mathfrak{H}^{0} .
$$

The finite double shuffle relations for MZVs are

$$
Z\left(w_{1} * w_{2}-w_{1} \amalg w_{2}\right)=0 \quad\left(w_{1}, w_{2} \in \mathfrak{H}^{0}\right) .
$$

The evaluation map is generalized in the following proposition.

Proposition 2.1 ([6]). We have two algebra homomorphisms

$$
Z^{*}: \mathfrak{H}_{*}^{1} \rightarrow \mathbb{R}[T] \quad \text { and } \quad Z^{\mathrm{II}}: \mathfrak{H}_{\mathrm{II}}^{1} \rightarrow \mathbb{R}[T]
$$

which are uniquely characterized by the property that they both extend the evaluation map $Z: \mathfrak{H}^{0} \rightarrow \mathbb{R}$ and send $y$ to $T$.

Then we have the extended double shuffle relations for MZVs.

Theorem $2.2([6])$. For any $w_{1} \in \mathfrak{H}^{1}$ and $w_{2} \in \mathfrak{H}^{0}$,

$$
Z^{*}\left(w_{1} \amalg w_{2}-w_{1} * w_{2}\right)=0 \quad \text { and } \quad Z^{\amalg}\left(w_{1} \amalg w_{2}-w_{1} * w_{2}\right)=0 .
$$


2.2. Algebraic setup of $N M Z V$ s. In this subsection, we introduce the algebraic setup of NMZVs. Define a $\mathbb{Q}$-linear map $\bar{Z}: \mathfrak{H}^{0} \rightarrow \mathbb{R}$ by

$$
\bar{Z}(1)=1 \quad \text { and } \quad \bar{Z}\left(z_{k_{1}} \cdots z_{k_{n}}\right)=\bar{\zeta}\left(k_{1}, \ldots, k_{n}\right) .
$$

We call this map the $n$-evaluation map. We next define the $n$-harmonic product $₹$ on $\mathfrak{H}^{1}$, which is the NMZV-counterpart of the harmonic product $*$, inductively by

$$
\begin{aligned}
1 \bar{*} w & =w \bar{*} 1=w, \\
z_{k} w_{1} \bar{*} z_{l} w_{2} & =z_{k}\left(w_{1} \bar{*} z_{l} w_{2}\right)+z_{l}\left(z_{k} w_{1} \bar{*} w_{2}\right)-z_{k+l}\left(w_{1} \bar{*} w_{2}\right)
\end{aligned}
$$

$\left(k, l \in \mathbb{Z}_{\geq 1}\right.$ and $w, w_{1}, w_{2}$ are words in $\left.\mathfrak{H}^{1}\right)$, extended by $\mathbb{Q}$-bilinearity. The $n$-harmonic product $\bar{*}$ has the following properties.

Proposition 2.3. The $n$-harmonic product $\bar{*}$ is commutative and associative.

Proof. We can prove this by induction (cf. Theorem 2.1 of [3]). But we give another proof later.

Proposition 2.3 says that $\mathfrak{H}^{1}$ with the product $\bar{*}$ has the structure of a commutative $\mathbb{Q}$-algebra. We denote this algebra by $\mathfrak{H}_{*}^{1}$. The subset $\mathfrak{H}^{0}$ is a subalgebra of $\mathfrak{H}^{1}$ with respect to $\bar{*}$ and we denote it by $\mathfrak{H}_{*}^{0}$.

We now introduce a $\mathbb{Q}$-linear map $S: \mathfrak{H}^{1} \rightarrow \mathfrak{H}^{1}$. Let $S_{1} \in \operatorname{Aut}(\mathfrak{H})$ be defined by $S_{1}(1)=1, S_{1}(x)=x$ and $S_{1}(y)=x+y$. Define the $\mathbb{Q}$-linear map $S: \mathfrak{H}^{1} \rightarrow \mathfrak{H}^{1}$ by

$$
S(1):=1 \quad \text { and } \quad S(F y):=S_{1}(F) y
$$

for all words $F \in \mathfrak{H}$. Then it is clear that $\bar{Z}=Z \circ S$ on $\mathfrak{H}^{0}$, i.e.,

$$
\bar{\zeta}\left(k_{1}, \ldots, k_{n}\right)=Z\left(S\left(z_{k_{1}} \cdots z_{k_{n}}\right)\right) \quad\left(k_{1} \geq 2\right) .
$$

For example, $\bar{\zeta}\left(k_{1}, k_{2}\right)=\zeta\left(k_{1}+k_{2}\right)+\zeta\left(k_{1}, k_{2}\right)=Z\left(S\left(z_{k_{1}} z_{k_{2}}\right)\right), \bar{\zeta}\left(k_{1}, k_{2}, k_{3}\right)=$ $\zeta\left(k_{1}+k_{2}+k_{3}\right)+\zeta\left(k_{1}+k_{2}, k_{3}\right)+\zeta\left(k_{1}, k_{2}+k_{3}\right)+\zeta\left(k_{1}, k_{2}, k_{3}\right)=Z\left(S\left(z_{k_{1}} z_{k_{2}} z_{k_{3}}\right)\right)$. As is clear from the definition of $S$, we also have the following relation:

$$
S\left(w_{1} w_{2}\right)=S_{1}\left(w_{1}\right) S\left(w_{2}\right) \quad\left(w_{1} \in \mathfrak{H}, w_{2} \in \mathfrak{H}^{1}\right) .
$$

Proposition 2.4. For $w_{1}, w_{2} \in \mathfrak{H}^{0}$,

$$
\bar{Z}\left(w_{1} \bar{*} w_{2}\right)=\bar{Z}\left(w_{1}\right) \bar{Z}\left(w_{2}\right) .
$$

This is also proved in [5] and [7]. To prove Proposition 2.4, we need the following lemma.

Lemma 2.5. Let $w, w_{1}, w_{2}$ be words $(\neq 1)$ in $\mathfrak{H}^{1}$ and $p, q$ positive integers. Then

$$
S\left(z_{p}\right) * S_{1}\left(z_{q}\right) w=S_{1}\left(z_{p} z_{q}\right) w+S_{1}\left(z_{q}\right)\left(S\left(z_{p}\right) * w\right)-S_{1}\left(z_{p+q}\right) w
$$


and

$$
\begin{aligned}
& S_{1}\left(z_{p}\right) w_{1} * S_{1}\left(z_{q}\right) w_{2} \\
= & S_{1}\left(z_{p}\right)\left(w_{1} * S_{1}\left(z_{q}\right) w_{2}\right)+S_{1}\left(z_{q}\right)\left(S_{1}\left(z_{p}\right) w_{1} * w_{2}\right)-S_{1}\left(z_{p+q}\right)\left(w_{1} * w_{2}\right) .
\end{aligned}
$$

Proof. We first prove (2.2). Put $w=z_{n} \widetilde{w}\left(n \geq 1, \widetilde{w} \in \mathfrak{H}^{1}\right)$. Then RHS of (2.2)

$$
\begin{aligned}
= & \left(x^{p-1} y+x^{p}\right)\left(x^{q-1} y+x^{q}\right) z_{n} \widetilde{w}+\left(x^{q-1} y+x^{q}\right)\left(z_{p} * z_{n} \widetilde{w}\right) \\
& -\left(x^{p+q-1} y+x^{p+q}\right) z_{n} \widetilde{w} \\
= & z_{p} z_{q} z_{n} \widetilde{w}+z_{p} z_{n+q} \widetilde{w}+z_{p+q} z_{n} \widetilde{w}+z_{n+p+q} \widetilde{w} \\
& +z_{q} z_{p} z_{n} \widetilde{w}+z_{q} z_{n}\left(z_{p} * \widetilde{w}\right)+z_{q} z_{n+p} \widetilde{w}+z_{p+q} z_{n} \widetilde{w} \\
& +z_{n+q}\left(z_{p} * \widetilde{w}\right)+z_{n+p+q} \widetilde{w}-z_{p+q} z_{n} \widetilde{w}-z_{n+p+q} \widetilde{w} \\
= & z_{p} * z_{q} z_{n} \widetilde{w}+z_{p} * z_{n+q} \widetilde{w}=S\left(z_{p}\right) * S_{1}\left(z_{q}\right) z_{n} \widetilde{w}=S\left(z_{p}\right) * S_{1}\left(z_{q}\right) w .
\end{aligned}
$$

Hence (2.2) follows. Putting $w_{1}=z_{m} \widetilde{w}_{1}, w_{2}=z_{n} \widetilde{w}_{2}\left(m, n \geq 1, \widetilde{w}_{1}, \widetilde{w}_{2} \in \mathfrak{H}^{1}\right)$, we can prove $(2.3)$ in the same way.

Proof of Proposition 2.4. It suffices to show that

$$
S\left(w_{1} \bar{*} w_{2}\right)=S\left(w_{1}\right) * S\left(w_{2}\right)
$$

for $w_{1}, w_{2} \in \mathfrak{H}^{1}$. We set $w_{1}=z_{p_{1}} \cdots z_{p_{m}}, w_{2}=z_{q_{1}} \cdots z_{q_{n}}$. We prove $(2.4)$ by induction on $m$. To ease the following calculation, we set $z_{\vec{p}}=z_{p_{2}} \cdots z_{p_{m}}$ and $z_{\vec{q}}=z_{q_{2}} \cdots z_{q_{n}}$.

(i) We prove the case $m=1$ by induction on $n$. When $n=1$, the assertion is immediate. We assume it is proven for $n-1$. Using (2.1), (2.2) and the induction hypothesis, we have

$$
\begin{aligned}
& S\left(z_{p_{1}} \bar{*} z_{q_{1}} z_{q_{2}} \cdots z_{q_{n}}\right)=S\left(z_{p_{1}} \bar{*} z_{q_{1}} z_{\vec{q}}\right) \\
& \quad=S\left(z_{p_{1}} z_{q_{1}} z_{\vec{q}}+z_{q_{1}}\left(z_{p_{1}} \bar{*} z_{\vec{q}}\right)-z_{p_{1}+q_{1}} z_{\vec{q}}\right) \\
& \quad=S_{1}\left(z_{p_{1}} z_{q_{1}}\right) S\left(z_{\vec{q}}\right)+S_{1}\left(z_{q_{1}}\right) S\left(z_{p_{1}} \bar{*} z_{\vec{q}}\right)-S_{1}\left(z_{p_{1}+q_{1}}\right) S\left(z_{\vec{q}}\right) \\
& \quad=S_{1}\left(z_{p_{1}} z_{q_{1}}\right) S\left(z_{\vec{q}}\right)+S_{1}\left(z_{q_{1}}\right)\left(S\left(z_{p_{1}}\right) * S\left(z_{\vec{q}}\right)\right)-S_{1}\left(z_{p_{1}+q_{1}}\right) S\left(z_{\vec{q}}\right) \\
& \quad=S\left(z_{p_{1}}\right) * S_{1}\left(z_{q_{1}}\right) S\left(z_{\vec{q}}\right)=S\left(z_{p_{1}}\right) * S\left(z_{q_{1}} z_{\vec{q}}\right)=S\left(z_{p_{1}}\right) * S\left(z_{q_{1}} z_{q_{2}} \cdots z_{q_{n}}\right) .
\end{aligned}
$$

(ii) We assume the assertion is proven for $m-1$. We prove it for $m$ by induction on $n$. When $n=1$, the assertion follows from (i) and the commutativity of $*, \bar{*}$. We assume it is true for $n-1$. Using (2.1), (2.3) and 
the induction hypothesis, we have

$$
\begin{aligned}
S( & \left(z_{p_{1}} z_{p_{2}} \cdots z_{p_{m}} \bar{*} z_{q_{1}} z_{q_{2}} \cdots z_{q_{n}}\right)=S\left(z_{p_{1}} z_{\vec{p}} z_{q_{1}} z_{\vec{q}}\right) \\
= & S\left(z_{p_{1}}\left(z_{\vec{p}} z_{q_{1}} z_{\vec{q}}\right)+z_{q_{1}}\left(z_{p_{1}} z_{\vec{p}} z_{\vec{q}}\right)-z_{p_{1}+q_{1}}\left(z_{\vec{p}} z_{\vec{q}}\right)\right) \\
= & S_{1}\left(z_{p_{1}}\right) S\left(z_{\vec{p}} z_{q_{1}} z_{\vec{q}}\right)+S_{1}\left(z_{q_{1}}\right) S\left(z_{p_{1}} z_{\vec{p}} z_{\vec{q}}\right)-S_{1}\left(z_{p_{1}+q_{1}}\right) S\left(z_{\vec{p}} * z_{\vec{q}}\right) \\
= & S_{1}\left(z_{p_{1}}\right)\left(S\left(z_{\vec{p}}\right) * S\left(z_{q_{1}} z_{\vec{q}}\right)\right)+S_{1}\left(z_{q_{1}}\right)\left(S\left(z_{p_{1}} z_{\vec{p}}\right) * S\left(z_{\vec{q}}\right)\right) \\
& -S_{1}\left(z_{p_{1}+q_{1}}\right)\left(S\left(z_{\vec{p}}\right) * S\left(z_{\vec{q}}\right)\right) \\
= & S_{1}\left(z_{p_{1}}\right)\left(S\left(z_{\vec{p}}\right) * S_{1}\left(z_{q_{1}}\right) S\left(z_{\vec{q}}\right)\right)+S_{1}\left(z_{q_{1}}\right)\left(S_{1}\left(z_{p_{1}}\right) S\left(z_{\vec{p}}\right) * S\left(z_{\vec{q}}\right)\right) \\
& -S_{1}\left(z_{p_{1}+q_{1}}\right)\left(S\left(z_{\vec{p}}\right) * S\left(z_{\vec{q}}\right)\right) \\
= & S_{1}\left(z_{p_{1}}\right) S\left(z_{\vec{p}}\right) * S_{1}\left(z_{q_{1}}\right) S\left(z_{\vec{q}}\right) \\
= & S\left(z_{p_{1}} z_{\vec{p}}\right) * S\left(z_{q_{1}} z_{\vec{q}}\right)=S\left(z_{p_{1}} z_{p_{2}} \cdots z_{p_{m}}\right) * S\left(z_{q_{1}} z_{q_{2}} \cdots z_{q_{n}}\right) .
\end{aligned}
$$

We now define the $n$-shuffle product $\bar{m}$ on $\mathfrak{H}$ which corresponds to the shuffle product $ш$. It is defined inductively by

$$
\begin{aligned}
1 \overline{\mathrm{I}} w= & w \overline{\mathrm{I}} 1=w, \\
u_{1} w_{1} \overline{\mathrm{\amalg}} u_{2} w_{2}= & u_{1}\left(w_{1} \overline{\mathrm{\amalg}} u_{2} w_{2}\right)+u_{2}\left(u_{1} w_{1} \overline{\mathrm{m}} w_{2}\right) \\
& -\delta\left(w_{1}\right) \tau\left(u_{1}\right) u_{2} w_{2}-\delta\left(w_{2}\right) \tau\left(u_{2}\right) u_{1} w_{1}
\end{aligned}
$$

$\left(u_{1}, u_{2} \in\{x, y\}\right.$ and $\left.w, w_{1}, w_{2} \in \mathfrak{H}\right)$, extended by $\mathbb{Q}$-bilinearity, where $\delta$ is defined by

$$
\delta(w)= \begin{cases}1 & (w=1) \\ 0 & (w \neq 1)\end{cases}
$$

for any word $w$, and $\tau$ is defined by $\tau(x)=y, \tau(y)=x$. The $n$-shuffle product has the following properties.

Proposition 2.6. The $n$-shuffle product is commutative and associative.

Proof. Let $w_{1}, w_{2}, w_{3} \in \mathfrak{H}$. We can check that $w_{1} \bar{\amalg} w_{2}=w_{2} \bar{\amalg} w_{1}$ by induction on $\left|w_{1}\right|+\left|w_{2}\right|$. We now prove $\left(w_{1} \overline{\mathrm{II}} w_{2}\right) \overline{\mathrm{I}} w_{3}=w_{1} \overline{\mathrm{II}}\left(w_{2} \overline{\mathrm{I}} w_{3}\right)$ by induction on $\left|w_{1}\right|+\left|w_{2}\right|+\left|w_{3}\right|$. The case $\left|w_{1}\right|+\left|w_{2}\right|+\left|w_{3}\right| \leq 2$ is obvious. Putting $w_{1}=u_{1} \widetilde{w}_{1}, w_{2}=u_{2} \widetilde{w}_{2}, w_{3}=u_{3} \widetilde{w}_{3}\left(u_{1}, u_{2}, u_{3} \in\{x, y\}\right)$, we have

$\left(w_{1} \overline{\mathrm{I}} w_{2}\right) \overline{\mathrm{m}} w_{3}$

$$
\begin{aligned}
& =u_{1}\left(\widetilde{w}_{1} \overline{\mathrm{m}} u_{2} \widetilde{w}_{2}\right) \overline{\mathrm{m}} u_{3} \widetilde{w}_{3}+u_{2}\left(u_{1} \widetilde{w}_{1} \overline{\mathrm{m}} \widetilde{w}_{2}\right) \overline{\mathrm{m}} u_{3} \widetilde{w}_{3} \\
& -\delta\left(\widetilde{w}_{1}\right) \tau\left(u_{1}\right) u_{2} \widetilde{w}_{2} \overline{\mathrm{m}} u_{3} \widetilde{w}_{3}-\delta\left(\widetilde{w}_{2}\right) \tau\left(u_{2}\right) u_{1} \widetilde{w}_{1} \overline{\mathrm{m}} u_{3} \widetilde{w}_{3} \\
& =u_{1}\left\{\left(\widetilde{w}_{1} \bar{\amalg} u_{2} \widetilde{w}_{2}\right) \bar{\amalg} u_{3} \widetilde{w}_{3}\right\}+u_{3}\left\{u_{1}\left(\widetilde{w}_{1} \bar{\amalg} u_{2} \widetilde{w}_{2}\right) \bar{\amalg} \widetilde{w}_{3}\right\} \\
& -\delta\left(\widetilde{w}_{3}\right) \tau\left(u_{3}\right) u_{1}\left(\widetilde{w}_{1} \bar{\amalg} u_{2} \widetilde{w}_{2}\right)+u_{2}\left\{\left(u_{1} \widetilde{w}_{1} \bar{\amalg} \widetilde{w}_{2}\right) \bar{\amalg} u_{3} \widetilde{w}_{3}\right\}
\end{aligned}
$$




$$
\begin{aligned}
& +u_{3}\left\{u_{2}\left(u_{1} \widetilde{w}_{1} \bar{\amalg} \widetilde{w}_{2}\right) \bar{\amalg} \widetilde{w}_{3}\right\}-\delta\left(\widetilde{w}_{3}\right) \tau\left(u_{3}\right) u_{2}\left(u_{1} \widetilde{w}_{1} \bar{\amalg} \widetilde{w}_{2}\right) \\
& -\delta\left(\widetilde{w}_{1}\right) \tau\left(u_{1}\right) u_{2} \widetilde{w}_{2} \bar{ш} u_{3} \widetilde{w}_{3}-\delta\left(\widetilde{w}_{2}\right) \tau\left(u_{2}\right) u_{1} \widetilde{w}_{1} \bar{ш} u_{3} \widetilde{w}_{3} \\
& =u_{1}\left\{\left(\widetilde{w}_{1} \bar{\amalg} u_{2} \widetilde{w}_{2}\right) \bar{\amalg} u_{3} \widetilde{w}_{3}\right\}+u_{2}\left\{\left(u_{1} \widetilde{w}_{1} \bar{\amalg} \widetilde{w}_{2}\right) \bar{\amalg} u_{3} \widetilde{w}_{3}\right\} \\
& +u_{3}\left\{\left(u_{1} \widetilde{w}_{1} \bar{\amalg} u_{2} \widetilde{w}_{2}\right) \bar{\amalg} \widetilde{w}_{3}\right\}-\delta\left(\widetilde{w}_{1}\right) \tau\left(u_{1}\right)\left(u_{2} \widetilde{w}_{2} \bar{\amalg} u_{3} \widetilde{w}_{3}\right) \\
& -\delta\left(\widetilde{w}_{2}\right) \tau\left(u_{2}\right)\left(u_{1} \widetilde{w}_{1} \overline{\mathrm{m}} u_{3} \widetilde{w}_{3}\right)-\delta\left(\widetilde{w}_{3}\right) \tau\left(u_{3}\right)\left(u_{1} \widetilde{w}_{1} \overline{\mathrm{m}} u_{2} \widetilde{w}_{2}\right) .
\end{aligned}
$$

In the last equality, we use the following three relations:

$u_{1}\left(\widetilde{w}_{1} \bar{\amalg} u_{2} \widetilde{w}_{2}\right)+u_{2}\left(u_{1} \widetilde{w}_{1} \bar{\amalg} \widetilde{w}_{2}\right)$

$=u_{1} \widetilde{w}_{1} \overline{\mathrm{II}} u_{2} \widetilde{w}_{2}+\delta\left(\widetilde{w}_{1}\right) \tau\left(u_{1}\right) u_{2} \widetilde{w}_{2}+\delta\left(\widetilde{w}_{2}\right) \tau\left(u_{2}\right) u_{1} \widetilde{w}_{1}$,

$\tau\left(u_{1}\right) u_{2} \widetilde{w}_{2} \bar{\amalg} u_{3} \widetilde{w}_{3}$

$=\tau\left(u_{1}\right)\left(u_{2} \widetilde{w}_{2} \bar{\amalg} u_{3} \widetilde{w}_{3}\right)+u_{3}\left(\tau\left(u_{1}\right) u_{2} \widetilde{w}_{2} \bar{\amalg} \widetilde{w}_{3}\right)-\delta\left(\widetilde{w}_{3}\right) \tau\left(u_{3}\right) \tau\left(u_{1}\right) u_{2} \widetilde{w}_{2}$, $\tau\left(u_{2}\right) u_{1} \widetilde{w}_{1} \bar{\amalg} u_{3} \widetilde{w}_{3}$

$=\tau\left(u_{2}\right)\left(u_{1} \widetilde{w}_{1} \bar{\amalg} u_{3} \widetilde{w}_{3}\right)+u_{3}\left(\tau\left(u_{2}\right) u_{1} \widetilde{w}_{1} \bar{\amalg} \widetilde{w}_{3}\right)-\delta\left(\widetilde{w}_{3}\right) \tau\left(u_{3}\right) \tau\left(u_{2}\right) u_{1} \widetilde{w}_{1}$.

On the other hand,

$w_{1} \bar{\amalg}\left(w_{2} \bar{\amalg} w_{3}\right)$

$$
\begin{aligned}
& =u_{1} \widetilde{w}_{1} \bar{\amalg} u_{2}\left(\widetilde{w}_{2} \bar{\amalg} u_{3} \widetilde{w}_{3}\right)+u_{1} \widetilde{w}_{1} \bar{\amalg} u_{3}\left(u_{2} \widetilde{w}_{2} \bar{\amalg} \widetilde{w}_{3}\right) \\
& -\delta\left(\widetilde{w}_{2}\right) u_{1} \widetilde{w}_{1} \overline{\mathrm{m}} \tau\left(u_{2}\right) u_{3} \widetilde{w}_{3}-\delta\left(\widetilde{w}_{3}\right) u_{1} \widetilde{w}_{1} \overline{\mathrm{m}} \tau\left(u_{3}\right) u_{2} \widetilde{w}_{2} \\
& =u_{1}\left\{\widetilde{w}_{1} \bar{\amalg} u_{2}\left(\widetilde{w}_{2} \bar{\amalg} u_{3} \widetilde{w}_{3}\right)\right\}+u_{2}\left\{u_{1} \widetilde{w}_{1} \bar{\amalg}\left(\widetilde{w}_{2} \bar{\amalg} u_{3} \widetilde{w}_{3}\right)\right\} \\
& -\delta\left(\widetilde{w}_{1}\right) \tau\left(u_{1}\right) u_{2}\left(\widetilde{w}_{2} \bar{\amalg} u_{3} \widetilde{w}_{3}\right)+u_{1}\left\{\widetilde{w}_{1} \overline{\bar{\omega}} u_{3}\left(u_{2} \widetilde{w}_{2} \overline{\bar{\omega}} \widetilde{w}_{3}\right)\right\} \\
& +u_{3}\left\{u_{1} \widetilde{w}_{1} \bar{\amalg}\left(u_{2} \widetilde{w}_{2} \bar{\amalg} \widetilde{w}_{3}\right)\right\}-\delta\left(\widetilde{w}_{1}\right) \tau\left(u_{1}\right) u_{3}\left(u_{2} \widetilde{w}_{2} \bar{\amalg} \widetilde{w}_{3}\right) \\
& -\delta\left(\widetilde{w}_{2}\right) u_{1} \widetilde{w}_{1} \bar{\amalg} \tau\left(u_{2}\right) u_{3} \widetilde{w}_{3}-\delta\left(\widetilde{w}_{3}\right) u_{1} \widetilde{w}_{1} \bar{\amalg} \tau\left(u_{3}\right) u_{2} \widetilde{w}_{2} \\
& =u_{1}\left\{\widetilde{w}_{1} \overline{\bar{\amalg}}\left(u_{2} \widetilde{w}_{2} \bar{\amalg} u_{3} \widetilde{w}_{3}\right)\right\}+u_{2}\left\{u_{1} \widetilde{w}_{1} \overline{\overline{ }}\left(\widetilde{w}_{2} \bar{\amalg} u_{3} \widetilde{w}_{3}\right)\right\} \\
& +u_{3}\left\{u_{1} \widetilde{w}_{1} \bar{\amalg}\left(u_{2} \widetilde{w}_{2} \bar{\amalg} \widetilde{w}_{3}\right)\right\}-\delta\left(\widetilde{w}_{1}\right) \tau\left(u_{1}\right)\left(u_{2} \widetilde{w}_{2} \bar{\amalg} u_{3} \widetilde{w}_{3}\right) \\
& -\delta\left(\widetilde{w}_{2}\right) \tau\left(u_{2}\right)\left(u_{1} \widetilde{w}_{1} \bar{\amalg} u_{3} \widetilde{w}_{3}\right)-\delta\left(\widetilde{w}_{3}\right) \tau\left(u_{3}\right)\left(u_{1} \widetilde{w}_{1} \bar{\amalg} u_{2} \widetilde{w}_{2}\right) \text {. }
\end{aligned}
$$

In the last equality, we use the following three relations:

$u_{2}\left(\widetilde{w}_{2} \bar{\amalg} u_{3} \widetilde{w}_{3}\right)+u_{3}\left(u_{2} \widetilde{w}_{2} \bar{\amalg} \widetilde{w}_{3}\right)$

$=u_{2} \widetilde{w}_{2} \bar{\amalg} u_{3} \widetilde{w}_{3}+\delta\left(\widetilde{w}_{2}\right) \tau\left(u_{2}\right) u_{3} \widetilde{w}_{3}+\delta\left(\widetilde{w}_{3}\right) \tau\left(u_{3}\right) u_{2} \widetilde{w}_{2}$,

$u_{1} \widetilde{w}_{1} \bar{\amalg} \tau\left(u_{2}\right) u_{3} \widetilde{w}_{3}$

$=u_{1}\left(\widetilde{w}_{1} \bar{\amalg} \tau\left(u_{2}\right) u_{3} \widetilde{w}_{3}\right)+\tau\left(u_{2}\right)\left(u_{1} \widetilde{w}_{1} \bar{ш} u_{3} \widetilde{w}_{3}\right)-\delta\left(\widetilde{w}_{1}\right) \tau\left(u_{1}\right) \tau\left(u_{2}\right) u_{3} \widetilde{w}_{3}$, $u_{1} \widetilde{w}_{1} \bar{\amalg} \tau\left(u_{3}\right) u_{2} \widetilde{w}_{2}$

$=u_{1}\left(\widetilde{w}_{1} \bar{\amalg} \tau\left(u_{3}\right) u_{2} \widetilde{w}_{2}\right)+\tau\left(u_{3}\right)\left(u_{1} \widetilde{w}_{1} \bar{\amalg} u_{2} \widetilde{w}_{2}\right)-\delta\left(\widetilde{w}_{1}\right) \tau\left(u_{1}\right) \tau\left(u_{3}\right) u_{2} \widetilde{w}_{2}$.

So we obtain the assertion by the induction hypothesis. 
Proposition 2.6 says that $\mathfrak{H}$ with the product $\overline{\mathrm{II}}$ has the structure of a commutative $\mathbb{Q}$-algebra. We denote it by $\mathfrak{H}_{\overline{\mathrm{II}}}$. The subsets $\mathfrak{H}^{1}$ and $\mathfrak{H}^{0}$ are subalgebras of $\mathfrak{H}$ with respect to $\overline{\overline{I I}}$ and we denote them by $\mathfrak{H}_{\overline{\mathrm{II}}}, \mathfrak{H}_{\overline{\mathrm{II}}}^{\frac{0}{1}}$ respectively.

Proposition 2.7. For $w_{1}, w_{2} \in \mathfrak{H}^{0}$,

$$
\bar{Z}\left(w_{1} \bar{\amalg} w_{2}\right)=\bar{Z}\left(w_{1}\right) \bar{Z}\left(w_{2}\right) .
$$

Proof. It suffices to prove that

$$
S\left(w_{1} \bar{\amalg} w_{2}\right)=S\left(w_{1}\right) \amalg S\left(w_{2}\right)
$$

for $w_{1}, w_{2} \in \mathfrak{H}^{1}$. We put $w_{1}=u_{1} u_{2} \cdots u_{m}$ and $w_{2}=v_{1} v_{2} \cdots v_{n}\left(u_{i}, v_{i} \in\right.$ $\{x, y\})$. We prove $(2.5)$ by induction on $m$. In order to simplify the proof, we set $u_{\vec{m}}:=u_{2} \cdots u_{m}$ and $v_{\vec{n}}:=v_{2} \cdots v_{n}$.

(i) We prove the case $m=1$ by induction on $n$. First,

$$
\begin{aligned}
S\left(u_{1} \bar{\amalg} v_{1}\right) & =S(y \bar{\amalg} y)=S\left(2 y^{2}-2 x y\right)=2(x+y) y-2 x y=2 y^{2} \\
& =y \amalg y=S(y) \amalg S(y)=S\left(u_{1}\right) \amalg S\left(v_{1}\right) .
\end{aligned}
$$

So the case $n=1$ is valid. We assume the assertion is proven for $n-1$. Using (2.1) and the induction hypothesis, we have

$$
\begin{aligned}
S\left(u_{1}\right. & \left.\overline{\mathrm{I}} v_{1} v_{2} \cdots v_{n}\right)=S\left(y \overline{\mathrm{m}} v_{1} v_{\vec{n}}\right) \\
& =S\left(y v_{1} v_{\vec{n}}+v_{1}\left(y \overline{\mathrm{m}} v_{\vec{n}}\right)-x v_{1} v_{\vec{n}}\right) \\
& =S_{1}(y) S_{1}\left(v_{1}\right) S\left(v_{\vec{n}}\right)+S_{1}\left(v_{1}\right) S\left(y \overline{\mathrm{m}} v_{\vec{n}}\right)-S_{1}(x) S_{1}\left(v_{1}\right) S\left(v_{\vec{n}}\right) \\
& =(x+y) S_{1}\left(v_{1}\right) S\left(v_{\vec{n}}\right)+S_{1}\left(v_{1}\right)\left(S(y) \amalg S\left(v_{\vec{n}}\right)\right)-x S_{1}\left(v_{1}\right) S\left(v_{\vec{n}}\right) \\
& =y S_{1}\left(v_{1}\right) S\left(v_{\vec{n}}\right)+S_{1}\left(v_{1}\right)\left(y \amalg S\left(v_{\vec{n}}\right)\right) \\
& =y \amalg S_{1}\left(v_{1}\right) S\left(v_{\vec{n}}\right)=S\left(u_{1}\right) \amalg\left(v_{1} v_{\vec{n}}\right)=S\left(u_{1}\right) \amalg S\left(v_{1} v_{2} \cdots v_{n}\right) .
\end{aligned}
$$

Thus we have the assertion for $n$.

(ii) We assume the assertion is proven for $m-1$. We prove it for $m$ by induction on $n$. The case $n=1$ is obvious by (i) and the commutativity of

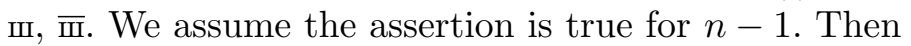

$$
\begin{aligned}
& S\left(u_{1} u_{2} \cdots u_{m} \overline{\mathrm{I}} v_{1} v_{2} \cdots v_{n}\right)=S\left(u_{1} u_{\vec{m}} \overline{\mathrm{m}} v_{1} v_{\vec{n}}\right) \\
& \quad=S\left(u_{1}\left(u_{\vec{m}} \overline{\mathrm{I}} v_{1} v_{\vec{n}}\right)+v_{1}\left(u_{1} u_{\vec{m}} \overline{\mathrm{I}} v_{\vec{n}}\right)\right) \\
& \quad=S_{1}\left(u_{1}\right) S\left(u_{\vec{m}} \overline{\mathrm{I}} v_{1} v_{\vec{n}}\right)+S_{1}\left(v_{1}\right) S\left(u_{1} u_{\vec{m}} \overline{\mathrm{I}} v_{\vec{n}}\right) \\
& \quad=S_{1}\left(u_{1}\right)\left(S\left(u_{\vec{m}}\right) \amalg S\left(v_{1} v_{\vec{n}}\right)\right)+S_{1}\left(v_{1}\right)\left(S\left(u_{1} u_{\vec{m}}\right) \amalg S\left(v_{\vec{n}}\right)\right) \\
& \quad=S_{1}\left(u_{1}\right)\left(S\left(u_{\vec{m}}\right) \amalg S_{1}\left(v_{1}\right) S\left(v_{\vec{n}}\right)\right)+S_{1}\left(v_{1}\right)\left(S_{1}\left(u_{1}\right) S\left(u_{\vec{m}}\right) \amalg S\left(v_{\vec{n}}\right)\right) \\
& \quad=S_{1}\left(u_{1}\right) S\left(u_{\vec{m}}\right) \amalg S_{1}\left(v_{1}\right) S\left(v_{\vec{n}}\right) \\
& \quad=S\left(u_{1} u_{\vec{m}}\right) \amalg S\left(v_{1} v_{\vec{n}}\right)=S\left(u_{1} u_{2} \cdots u_{m}\right) \amalg S\left(v_{1} v_{2} \cdots v_{n}\right) .
\end{aligned}
$$


Because the $n$-evaluation map $\bar{Z}$ is a homomorphism with respect to $\bar{*}$ and $\overline{\mathrm{II}}$, we have the following theorem.

TheOREM 2.8 (Finite double shuffle relations for NMZVs). For $w_{1}, w_{2}$ $\in \mathfrak{H}^{0}$,

$$
\bar{Z}\left(w_{1} \mp w_{2}-w_{1} \bar{\amalg} w_{2}\right)=0 .
$$

2.3. Extended double shuffle relations for NMZVs. In this subsection, we generalize Theorem 2.8. In the following lemma, we introduce the inverse of $S$.

LEMMA 2.9.

(i) Define $S_{2} \in \mathfrak{H}$ by $S_{2}(1)=1, S_{2}(x)=x$ and $S_{2}(y)=y-x$, and define the $\mathbb{Q}$-linear map $\widetilde{S}: \mathfrak{H}^{1} \rightarrow \mathfrak{H}^{1}$ by

$$
\widetilde{S}(1)=1 \quad \text { and } \quad \widetilde{S}(F y):=S_{2}(F) y
$$

for $F \in \mathfrak{H}$. Then $\widetilde{S} \circ S=S \circ \widetilde{S}=$ id on $\mathfrak{H}^{1}$.

(ii) For $w_{1}, w_{2} \in \mathfrak{H}^{1}$,

$$
\widetilde{S}\left(w_{1} * w_{2}\right)=\widetilde{S}\left(w_{1}\right) \approx \widetilde{S}\left(w_{2}\right), \quad \widetilde{S}\left(w_{1} ш w_{2}\right)=\widetilde{S}\left(w_{1}\right) \bar{\amalg} \widetilde{S}\left(w_{2}\right) .
$$

Proof. (i) By definition, we have $\widetilde{S} \circ S(1)=S \circ \widetilde{S}(1)=1$. Let $w \in \mathfrak{H}^{1} \backslash\{1\}$. Then we can write $w=w_{1} y\left(w_{1} \in \mathfrak{H}\right)$, and we have

$$
\begin{aligned}
& \widetilde{S} \circ S(w)=\widetilde{S} \circ S\left(w_{1} y\right)=\widetilde{S}\left(S_{1}\left(w_{1}\right) y\right)=S_{2}\left(S_{1}\left(w_{1}\right)\right) y=w_{1} y=w, \\
& S \circ \widetilde{S}(w)=S \circ \widetilde{S}\left(w_{1} y\right)=S\left(S_{2}\left(w_{1}\right) y\right)=S_{1}\left(S_{2}\left(w_{1}\right)\right) y=w_{1} y=w .
\end{aligned}
$$

This proves (i); and (ii) is clear from (2.4), (2.5) and (i).

By Lemma 2.9 (i), we can denote $\widetilde{S}$ by $S^{-1}$. Then Lemma 2.9 (ii) can be restated as follows:

$$
\begin{aligned}
S^{-1}\left(w_{1} * w_{2}\right) & =S^{-1}\left(w_{1}\right) \bar{*} S^{-1}\left(w_{2}\right), \\
S^{-1}\left(w_{1} \amalg w_{2}\right) & =S^{-1}\left(w_{1}\right) \bar{\amalg} S^{-1}\left(w_{2}\right) .
\end{aligned}
$$

Using (2.6), we give the proof of Proposition 2.3.

Proof of Proposition 2.3. By using (2.6) and the commutativity of the harmonic product $*$, we have

$$
\begin{aligned}
w_{1} \bar{*} w_{2} & =S^{-1}\left(S\left(w_{1}\right)\right) \star S^{-1}\left(S\left(w_{2}\right)\right)=S^{-1}\left(S\left(w_{1}\right) * S\left(w_{2}\right)\right) \\
& =S^{-1}\left(S\left(w_{2}\right) * S\left(w_{1}\right)\right)=S^{-1}\left(S\left(w_{2}\right)\right) S^{-1}\left(S\left(w_{1}\right)\right)=w_{2} \bar{*} w_{1} .
\end{aligned}
$$

So the $n$-harmonic product $₹$ is commutative. We next prove its associativity by using (2.6) and the associativity of $*$ :

$$
\begin{aligned}
w_{1} \bar{*}\left(w_{2} \bar{*} w_{3}\right) & =S^{-1}\left(S\left(w_{1}\right)\right) \bar{*}\left(S^{-1}\left(S\left(w_{2}\right)\right) S^{-1}\left(S\left(w_{3}\right)\right)\right) \\
& =S^{-1}\left(S\left(w_{1}\right)\right) \bar{*} S^{-1}\left(S\left(w_{2}\right) * S\left(w_{3}\right)\right) \\
& =S^{-1}\left(S\left(w_{1}\right) *\left(S\left(w_{2}\right) * S\left(w_{3}\right)\right)\right)
\end{aligned}
$$




$$
\begin{aligned}
& =S^{-1}\left(\left(S\left(w_{1}\right) * S\left(w_{2}\right)\right) * S\left(w_{3}\right)\right) \\
& =S^{-1}\left(S\left(w_{1}\right) * S\left(w_{2}\right)\right) \bar{*} S^{-1}\left(S\left(w_{3}\right)\right) \\
& =\left(S^{-1}\left(S\left(w_{1}\right)\right) \bar{*} S^{-1}\left(S\left(w_{2}\right)\right)\right) \bar{*} w_{3}=\left(w_{1} \bar{*} w_{2}\right) \bar{*} w_{3} .
\end{aligned}
$$

Lemma 2.10. Let $\circ=*$ or ш. $A$ word $y^{m} w\left(m \geq 0, w \in \mathfrak{H}^{0}\right)$ in $\mathfrak{H}^{1}$ is uniquely represented as

$$
y^{m} w=w_{0}+w_{1} \bar{\circ} y+w_{2} \bar{\circ} y^{\bar{\circ} 2}+\cdots+w_{m} \bar{\circ} y^{\bar{\circ} m} \quad\left(w_{i} \in \mathfrak{H}^{0}\right),
$$

i.e., $\mathfrak{H}_{\overline{0}}^{0}[y] \simeq \mathfrak{H}_{\overline{0}}^{1}$.

Proof. We first prove that $y^{m} w$ can be represented as in (2.8). By Corollary 5 of [6], we have

$$
(y+x)^{m} S(w)=\sum_{i=0}^{m} v_{i} \circ y^{\circ i} \quad\left(v_{i} \in \mathfrak{H}^{0}\right) .
$$

Using (2.6) or (2.7), we obtain

$$
y^{m} w=\sum_{i=0}^{m} S^{-1}\left(v_{i}\right) \bar{\circ} y^{\bar{o} i} .
$$

(We have $S^{-1}\left(w_{1} w_{2}\right)=S_{2}\left(w_{1}\right) S^{-1}\left(w_{2}\right)$ for $w_{1} \in \mathfrak{H}, w_{2} \in \mathfrak{H}^{1}$.) Therefore, the representation (2.8) follows from $S^{-1}\left(\mathfrak{H}^{0}\right) \subset \mathfrak{H}^{0}$. We next prove the uniqueness of this representation. Suppose that

$$
\sum_{i=0}^{m} w_{i} \bar{\circ} y^{\bar{\circ} i}=\sum_{i=0}^{m} v_{i} \bar{\circ} y^{\bar{\circ} i} \quad\left(w_{i}, v_{i} \in \mathfrak{H}^{0}\right) \text {. }
$$

Using (2.4) or (2.5), we have

$$
\sum_{i=0}^{m} S\left(w_{i}\right) \circ y^{\circ i}=\sum_{i=0}^{m} S\left(v_{i}\right) \circ y^{\circ i} .
$$

As $\mathfrak{H}_{\circ}^{0}[y] \simeq \mathfrak{H}_{\circ}^{1}$ (see $[3]$ and $\left.[10]\right)$, we have $S\left(w_{i}\right)=S\left(v_{i}\right)$ for $i=0,1, \ldots, m$. This yields the desired uniqueness.

Proposition 2.11. We have two algebra homomorphisms

$$
\bar{Z}^{\bar{*}}: \mathfrak{H}_{\bar{*}}^{1} \rightarrow \mathbb{R}[T] \quad \text { and } \quad \bar{Z}^{\overline{\mathrm{W}}}: \mathfrak{H}_{\overline{\mathrm{W}}}^{1} \rightarrow \mathbb{R}[T]
$$

which are uniquely characterized by the property that they both extend the $n$-evaluation map $\bar{Z}: \mathfrak{H}^{0} \rightarrow \mathbb{R}$ and send $y$ to $T$.

Proof. The assertion follows because $\bar{Z}$ is a homomorphism with respect to $\bar{*}, \overline{\mathrm{II}}$ and we have isomorphisms $\mathfrak{H}_{\bar{*}}^{0}[y] \simeq \mathfrak{H}_{\bar{*}}^{1}, \mathfrak{H}_{\overline{\mathrm{II}}}^{0}[y] \simeq \mathfrak{H}_{\overline{\mathrm{II}}}^{1}$.

The $\mathbb{Q}$-algebra homomorphisms $\bar{Z}^{\bar{*}}, \bar{Z}^{\overline{\mathrm{I}}}$ satisfy the following relations:

$$
\bar{Z}^{\bar{*}}=Z^{*} \circ S, \quad \overline{Z^{\bar{\amalg}}}=Z^{\amalg} \circ S
$$


( $\circ$ means composition). Indeed, $Z^{*} \circ S$ and $Z^{\amalg} \circ S$ satisfy the conditions of Proposition 2.11.

TheOREM 2.12 (Extended double shuffle relations for NMZVs). For $w_{1} \in \mathfrak{H}^{1}$ and $w_{2} \in \mathfrak{H}^{0}$,

$\bar{Z}^{\bar{*}}\left(w_{1} \overline{\mathrm{I}} w_{2}-w_{1} \bar{*} w_{2}\right)=0 \quad$ and $\quad \overline{Z^{\overline{\mathrm{W}}}}\left(w_{1} \overline{\mathrm{m}} w_{2}-w_{1} \bar{*} w_{2}\right)=0$.

Proof. By using (2.4), (2.5) and the relation $\bar{Z}^{*}=Z^{*} \circ S$, we have

$\bar{Z}^{\bar{*}}\left(w_{1} \bar{\amalg} w_{0}-w_{1} \bar{*} w_{0}\right)=Z^{*} \circ S\left(w_{1} \bar{\amalg} w_{0}-w_{1} \bar{*} w_{0}\right)$

$$
=Z^{*}\left(S\left(w_{1}\right) \amalg S\left(w_{0}\right)-S\left(w_{1}\right) * S\left(w_{0}\right)\right)=0 .
$$

The last equality follows from Theorem 2.2 and the inclusions $S\left(\mathfrak{H}^{1}\right) \subset \mathfrak{H}^{1}$ and $S\left(\mathfrak{H}^{0}\right) \subset \mathfrak{H}^{0}$. The other identity can be proven in the same way.

3. Application. In [2], Hoffman proved the following theorem.

Theorem 3.1 ([2]). For positive integers $k_{1}, \ldots, k_{n}$ with $k_{1} \geq 2$,

$$
\begin{aligned}
\sum_{i=1}^{n} \zeta\left(k_{1}, \ldots, k_{i-1}, k_{i}+1, k_{i+1}, \ldots, k_{n}\right) & \\
= & \sum_{\substack{1 \leq i \leq n \\
k_{i} \geq 2}} \sum_{j=0}^{k_{i}-2} \zeta\left(k_{1}, \ldots, k_{i-1}, k_{i}-j, j+1, k_{i+1}, \ldots, k_{n}\right) .
\end{aligned}
$$

In this section, we prove an analogue of Hoffman's relations for NMZVs:

TheOREM 3.2. For positive integers $k_{1}, \ldots, k_{n}$ with $k_{1} \geq 2$,

$$
\begin{aligned}
\sum_{i=1}^{n}\left(k_{i}-1+\delta_{n i}\right) \bar{\zeta}\left(k_{1}, \ldots, k_{i-1}, k_{i}+1, k_{i+1}, \ldots, k_{n}\right) \\
=\sum_{\substack{1 \leq i \leq n \\
k_{i} \geq 2}} \sum_{j=0}^{k_{i}-2} \bar{\zeta}\left(k_{1}, \ldots, k_{i-1}, k_{i}-j, j+1, k_{i+1}, \ldots, k_{n}\right) .
\end{aligned}
$$

We first prove the following lemma.

LEMMA 3.3 .

(i) Let $w \in \mathfrak{H}$ and let $k$ be a positive integer. Then

$$
y \overline{\mathrm{I}} z_{k} w= \begin{cases}z_{1} z_{k}+\sum_{j=0}^{k-2} z_{k-j} z_{j+1}-(k+1) z_{k+1}+z_{k} z_{1} & (w=1), \\ z_{1} z_{k} w+\sum_{j=0}^{k-2} z_{k-j} z_{j+1} w-k z_{k+1} w+z_{k}(y \overline{\mathrm{I}} w) & (w \neq 1),\end{cases}
$$

where the summation is treated as 0 when $k=1$. 
(ii) For $k_{1}, \ldots, k_{n} \in \mathbb{Z}_{\geq 1}$,

$$
\begin{aligned}
y \overline{\mathrm{II}} z_{k_{1}} \cdots z_{k_{n}}= & \sum_{i=0}^{n} z_{k_{1}} \cdots z_{k_{i}} z_{1} z_{k_{i+1}} \cdots z_{k_{n}} \\
& +\sum_{\substack{1 \leq i \leq n \\
k_{i} \geq 2}} \sum_{j=0}^{k_{i}-2} z_{k_{1}} \cdots z_{k_{i-1}} z_{k_{i}-j} z_{j+1} z_{k_{i+1}} \cdots z_{k_{n}} \\
& -\sum_{\substack{i=1 \\
n}}^{n}\left(k_{i}+\delta_{n i}\right) z_{k_{1}} \cdots z_{k_{i-1}} z_{k_{i}+1} z_{k_{i+1}} \cdots z_{k_{n}} .
\end{aligned}
$$

Proof. (i) The case $k=1$ is clear from the definition of $\bar{\varpi}$, and the case $k \geq 2$ can be proved by induction on $k$.

(ii) We prove the assertion by induction on $n$. The case $n=1$ follows from (i). We assume that the assertion is true for $n-1$. Using (i), we obtain

$$
\begin{aligned}
y \overline{\mathrm{II}} z_{k_{1}} z_{k_{2}} \cdots z_{k_{n}}= & z_{1} z_{k_{1}} z_{k_{2}} \cdots z_{k_{n}}+\sum_{j=0}^{k_{1}-2} z_{k_{1}-j} z_{j+1} z_{k_{2}} \cdots z_{k_{n}} \\
& -k_{1} z_{k_{1}+1} z_{k_{2}} \cdots z_{k_{n}}+z_{k_{1}}\left(y \overline{\mathrm{m}} z_{k_{2}} \cdots z_{k_{n}}\right) .
\end{aligned}
$$

By the induction hypothesis, this equals the right hand side of (3.1).

Proof of Theorem 3.2. By Lemma 3.3 and the definition of $\bar{*}$, we have $y \overline{\mathrm{I}} z_{k_{1}} z_{k_{2}} \cdots z_{k_{n}}-y \bar{*} z_{k_{1}} z_{k_{2}} \cdots z_{k_{n}}$

$$
\begin{aligned}
= & \sum_{\substack{1 \leq i \leq n \\
k_{i} \geq 2}} \sum_{j=0}^{k_{i}-2} z_{k_{1}} \cdots z_{k_{i-1}} z_{k_{i}-j} z_{j+1} z_{k_{i+1}} \cdots z_{k_{n}} \\
& -\sum_{i=1}^{n}\left(k_{i}+\delta_{n i}-1\right) z_{k_{1}} \cdots z_{k_{i-1}} z_{k_{i}+1} z_{k_{i+1}} \cdots z_{k_{n}} .
\end{aligned}
$$

The right hand side is in $\mathfrak{H}^{0}$ as $k_{1} \geq 2$. Therefore, the assertion follows from Theorem 2.12.

\section{References}

[1] T. Aoki and Y. Ohno, Sum relations for multiple zeta values and connection formulas for the Gauss hypergeometric functions, Publ. Res. Inst. Math. Sci. 41 (2005), 329337.

[2] M. Hoffman, Multiple harmonic series, Pacific J. Math. 152 (1992), 275-290.

[3] —, The algebra of multiple harmonic series, J. Algebra 194 (1997), 477-495.

[4] M. Hoffman and Y. Ohno, Relations of multiple zeta values and their algebraic expression, ibid. 262 (2003), 332-347.

[5] K. Ihara, J. Kajikawa, Y. Ohno and J. Okuda, MZSVs vs. MZVs, in preparation. 
[6] K. Ihara, M. Kaneko and D. Zagier, Derivation and double shuffle relations for multiple zeta values, Compos. Math. 142 (2006), 307-338.

[7] G. Kawashima, A class of relations among multiple zeta values, preprint, arXiv: math.NT/0702824.

[8] Y. Ohno, A generalization of the duality and sum formulas on the multiple zeta values, J. Number Theory 74 (1999), 39-43.

[9] Y. Ohno and N. Wakabayashi, Cyclic sum of multiple zeta values, Acta Arith. 123 (2006), 289-295.

[10] C. Reutenauer, Free Lie Algebras, Oxford Sci. Publ., Oxford Univ. Press, 1993.

Graduate School of Mathematics

Kyushu University

Fukuoka 812-8581, Japan

E-mail: muneta@math.kyushu-u.ac.jp

Received on 23.10.2007

and in revised form on 8.9.2008 